\title{
Publisher Correction: Glial responses to implanted electrodes in the brain
}

Joseph W. Salatino (iD, Kip A. Ludwig, Takashi D. Y. Kozai and Erin K. Purcell

Correction to: Nature Biomedical Engineering 1, 862-877 (2017); published online 10 November 2017.

In the version of this Review Article originally published, in Fig. 4b, the label 'Glutamate' was mistakenly duplicated and an arrow between a purinergic P2 receptor and a glutamate transporter was missing. The figure has now been updated in all versions of the Review Article. 